\title{
Subcarrier Allocation in Multiuser OFDM Systems: Complexity and Approximability
}

\author{
Pai-Han Huang, Yi Gai and Bhaskar Krishnamachari \\ School of Electrical Engineering \\ University of Southern California \\ Los Angeles, California 90089 \\ Email: \{paihanhu, ygai, bkrishna\}@usc.edu
}

\author{
Ashwin Sridharan \\ Sprint Applied Research and Advanced Technology Labs \\ California, USA \\ Ashwin.Sridharan@sprint.com
}

\begin{abstract}
We consider a number of related problem formulations pertaining to adaptive subcarrier allocation in multiuser Orthogonal Frequency-Division Multiplexing (OFDM) systems, and prove that they are NP-hard. Thus there exist no known algorithms that can provide optimal solutions for all instances of these problems in polynomial time. We further prove that these problems are hard to approximate in polynomial time. Finally, we discuss qualitatively the settings under which worstcase performance is likely to be observed.
\end{abstract}

\section{INTRODUCTION}

In multiuser OFDM systems, the frequency band is divided into a large number of small bands called subcarriers that use specific frequencies so as to be completely orthogonal to each other. In every time-slot, each user is assigned a disjoint set of subcarriers across which the user may spread information for transmission purposes. Because of its capability of exploiting multi-path fading and spatial/temporal/user diversity to improve performance, OFDM has become the physical layer transmission scheme of choice adopted by fourth generation wireless networks, e.g. cellular networks [1], broadband LANs [2], to provide high-speed mobile wireless data services.

It has been shown that dynamic subcarrier allocation to users that utilizes knowledge of channel conditions can significantly improve system performance. This is a combinatorial resource allocation problem: in each time-slot, given $m$ users, $n$ sub-carriers and complete channel knowledge, how to assign disjoint sets of subcarriers to each user so as to optimize some system objective. The allocation problem has received active interest in the research community and has been studied from two perspectives: schemes that seek to minimize the amount of transmit power (known as Margin-Adaptive (MA) approaches [3][4][5][6]) and those that seek to maximize throughput (known as Rate-Adaptive (RA) approaches [7][8][5]). Although optimal/near-optimal solutions for single user OFDM that use water-filling [9] are well known, optimal resource allocation in multiuser OFDM is still under-explored. A common theme (elaborated in Section II) across previous works on this topic is that they propose heuristics that are evaluated primarily through simulations. While such results show heuristics performing under particular simulated settings, it is important (from both academic and practical perspectives) to know under what scenarios such heuristics (or indeed any polynomial time algorithm) may not perform well.

We address this gap in the existing literature by formally investigating the complexity of subcarrier allocation under several scenarios, for both power minimization (MA) and rate maximization (RA). A mathematical formulation of MA and RA can be found in [5], while in some other papers, slightly different objectives from the definitions in [5] are adopted. In this work, we consider two different objectives for power minimization: (1) minimize overall power consumption (MinMA), and (2) minimize the maximal user power consumption (MinMax-MA), as well as two different goals for rate maximization: (1) maximize overall transmission rate (MaxRA), and (2) maximize the minimal user transmission rate (MaxMin-RA).

Our contributions can be summarized as follows :

1) We prove that all these problems, i.e. Min-MA, MinMax-MA, Max-RA, and MaxMin-RA, are NP-hard, thus it is impossible to guarantee optimality for any polynomial time solutions, unless $P=N P^{1}$.

2) We prove it is NP-hard to approximate Min-MA and MinMax-MA problems within factor $\alpha, \forall \alpha>1$. In other words, it is impossible for any polynomial time algorithm to have a guaranteed performance within a constant-factor of the optimal in the worst case, unless $P=N P$.

3) For Max-RA and MaxMin-RA, we show that achieving an approximation factor of $\frac{1}{m-1}$ is NP-hard, where $m$ is the number of subcarriers.

4) We qualitatively identify the worst-case situations where heuristics are likely to yield poor quality solutions.

This paper is organized as follows: In Section II we outline past related work and differentiate our contributions, system models and constraints. Section III presents the system model, formal problem formulation, and NP-hardness proof. In Section IV, we prove the in-approximability for powerminimization version and hard-to-approximate ratio for ratemaximization version of our problem. In Section V, we discuss qualitatively the conditions under which the worstcase scenarios may arise. Future directions are addressed in Section VI.

\footnotetext{
${ }^{1}$ If $P=N P$, then a problem is as easy to compute as to verify it. Consequently, for all the well-known hard NP-complete problems, such as Traveling Salesman, there would would exist an algorithm which can guarantee optimal solutions in polynomial computation time. It is generally conjectured that $P \neq N P$. For more details on NP-hardness, see [10].
} 


\section{RELATED WORK}

Wong et al. [4] propose a multiuser, multi-subcarrier, bit and power allocation algorithm, which aims to minimize the overall power consumption. In their paper, no subcarrier can be assigned to more than one user, and users are allowed to use different modulation schemes on different subcarriers allocated to themselves. The authors adopt a greedy bit loading algorithm for the single user, multi-subcarrier case, and propose an iterative search algorithm exploits Lagrangian Relaxation technique to deal with user-subcarrier assignment issues. However, this algorithm does not converge rapidly in general. We refer to their problem formulation in this paper to as Min-MA. Rhee et al. [11] try to maximize the minimal user capacity, such that a fixed total power budget is given, and we refer to this problem as MaxMin-RA. This problem can be optimally solved when a subcarrier is allowed to be shared by multiple users, and the authors propose a suboptimal algorithm in which every subcarrier is allocated with equal power. Jang et al. [12] try to maximize overall transmission rate under power constraints. Although the authors allow multiple users to share a subcarrier, they prove that the system objective can only be achieved when every subcarrier is exclusively occupied. We refer to their problem formulation as Max-RA. Kivanc et al. [3] adopt a similar formulation as [4]. The authors propose a two-step solution: first determine the number of subcarriers allocated to each user, and then allocate subcarriers to users in a greedy manner. Thereafter, they refine their solution quality by using a local search technique. Kim et al. [5] try to solve both RA and MA problems with formulations similar to [11] and [4], respectively. The authors demonstrate the integer linear programming (ILP) formulation of both problems, and propose two heuristics. Although using ILP can optimally solve both problems, it is known that the ILP computing cost grows exponentially with instance size. By assuming that every subcarrier allocated to the same user is loaded with same amount of bit, the first heuristic uses integer relaxation technique to transform the original ILP to a LP, and the other one is an application of Vogel's method [13]. Abrardo et al. [6] study both single and multi cells versions of the margin adaptive problem. By assuming that only one transmission format is available to all users, Alen et al. [14] devise a distributed algorithm aims to maximize system capacity. They first divide the available subcarriers into a set of partitions, and let users contend for these partitions among themselves. To solve the issue of partition sharing, i.e. multiple users access the same partition, their solution includes a conflict resolution mechanism.

To sum up, a rigorous algorithmic hardness proof of MinMA, MinMax-MA, Max-RA, and MaxMin-RA is missing in existing works. All optimal solutions proposed in existing publications are not guaranteed to be polynomial time, e.g. the ILP formulation in [5] and [11]. Although some special cases do have clean polynomial time solutions (e.g. [6] deals with Min-MA under the assumption that only one transmission format is available to all users) they are not optimal for the general case because of the restrictions imposed. In addition, all heuristics which aim to solve the general case of MinMA, MinMax-MA, Max-RA, and MaxMin-RA have been verified by simulation only, and none of them has a provable performance bound.

\section{NP-HARDNESS OF SUBCARRIER ALLOCATION PROBLEMS}

\section{A. Problem Descriptions}

The symbols used in the following equations are summarized in Table I.

Mathematically, we can formulate Min-MA as equation 1:

$$
\begin{array}{cl}
\operatorname{minimize} & \sum_{u_{i} \in U} \sum_{s_{j} \in S} f_{i j}\left(r_{i j} d_{i j}\right) \\
\text { subject to: } \quad & \sum_{s_{j} \in S} r_{i j} d_{i j} \geq r_{i}, \forall u_{i} \in U \\
& \sum_{u_{i} \in U} d_{i j} \leq 1, \forall s_{j} \in S \\
& r_{i j} \in T_{j}, \forall u_{i} \in U, s_{j} \in S \\
& \sum_{s_{j} \in S} d_{i j} \geq 1, \forall u_{i} \in U \\
& d_{i j} \in\{0,1\}, \forall u_{i} \in U, s_{j} \in S
\end{array}
$$

The formulation of Min-MA can be stated by replacing the objective with the following one:

$$
\text { minimize } \max _{u_{i} \in U}\left\{\sum_{s_{j} \in S} f_{i j}\left(r_{i j} d_{i j}\right)\right\}
$$

Max-RA can be described as equation 2:

$$
\begin{array}{ll}
\text { maximize } & \sum_{u_{i} \in U} \sum_{s_{j} \in S} r_{i j} d_{i j} \\
\text { subject to: } \quad & \sum_{u_{i} \in U} d_{i j} \leq 1, \forall s_{j} \in S \\
& \sum_{s_{j} \in S} d_{i j} \geq 1, \forall u_{i} \in U \\
& d_{i j} \in\{0,1\}, \forall u_{i} \in U, s_{j} \in S \\
& \sum_{u_{i} \in U} \sum_{s_{j} \in S} f_{i j}\left(r_{i j} d_{i j}\right) \leq P
\end{array}
$$

Similarly, MaxMin-RA can be stated by replacing the objective in equation 2 with the following one:

$$
\operatorname{maximize} \min _{u_{i} \in U}\left\{\sum_{s_{j} \in S} r_{i j} d_{i, j}\right\}
$$

\section{B. Proof Strategy}

The proof strategy uses a polynomial number of steps to transform an arbitrary instance of a well known NPhard problem to a special case of the problems we are interested in, i.e. Min-MA, MinMax-MA, Max-RA, MaxMinRA. are interested in, i.e. Min-MA, MinMax-MA, Max-RA, and MaxMin-RA. The process of transforming one instance to another instance in polynomial time is called polynomial reduction. If there exists a polynomial time algorithm that can optimally solve the problem we are interested in, say Min-MA, then we can always solve the original well known NP-hard problem in polynomial time by transforming the solutions provided the algorithm designed for Min-RA, which contradicts the fact that an NP-hard problem cannot be solved optimally in polynomial time unless $\mathrm{P}=\mathrm{NP}$ [10]. 
TABLE I

DEFINITION OF SYMBOLS

\begin{tabular}{|c||l|}
\hline \multicolumn{1}{|c||}{$S$} & The set of all subcarriers. $S=\left\{s_{i} \mid i=1, \ldots, m\right\}$. \\
\hline$U$ & The set of all users. $U=\left\{u_{i} \mid i=1, \ldots, n\right\}$. \\
\hline$r_{i}$ & Transmit rate request of user $i$. \\
\hline$r_{i j}$ & The transmit rate user $i$ loads on subcarrier $j$. \\
\hline$P$ & Overall power budget of the system. \\
\hline$S^{i}$ & The set of subcarriers that are allocated to user $i$. \\
\hline$f_{i j}\left(r_{i j}\right)$ & The power for user $i$ to transmit at rate $r_{i j}$ on subcarrier $j$ is $f_{i j}\left(r_{i j}\right)$. \\
\hline$d_{i j}$ & $d_{i j}=1$ if sub-carrier $j$ is allocated to user $i$. Otherwise, $d_{i j}=0$. \\
\hline$T_{i}$ & The set of feasible rates allowed on subcarrier $i$. \\
\hline
\end{tabular}

\section{NP-hardness Proof}

We plan to prove the NP-hardness of Min-RA by doing polynomial reduction from Subset Sum, which is a wellknown NP-hard problem and can be defined as follows:

Definition 1: Subset Sum Given a set of natural numbers $W=\left\{w_{1}, w_{2}, \ldots\right\}$, and a positive integer $V<\sum_{w_{i} \in W} w_{i}$, does there exist a subset $S \in W$, such that the sum of $S$ equals to $V$ ?

When we say a Subset Sum instance is satisfiable, it means there exists a subset $S \in W$ such that its sum equals to $V$. If such subset $S$ does not exist, we say the input instance cannot be satisfied. Note that, the answer to Subset Sum is simply YES or NO.

We refer to the mathematical definition of Min-MA in (1) as the optimization version of Min-MA, we define the decision version of Min-MA as follows:

Problem 1: Given a set of $n$ user rate requests, nondecreasing rate-power functions ${ }^{2}$ for $n$ users and $m$ subcarriers combination, and $m>n$, does there exist a set of subcarrier and user assignment with rate allocation on individual subcarrier, such that the sum of all user's power consumption is at most $P$, every user's request is satisfied, and no subcarrier is allocated to more than 1 user?

Although the answers to the optimization version and decision version of Min-MA are different (While in the former version, we need specific values for each variables, the answer to the later case is only YES or NO.), they have the same hardness in terms of computational complexity[10]. Therefore, if it is not stated specifically, all problems in the following contents refer to the decision version.

Theorem 1: Min-MA is NP-hard.

Proof: We first demonstrate how to transform an arbitrary instance of Subset Sum to a special instance of Min-MA in polynomial time. Consider an arbitrary instance of Subset Sum, with a set of natural numbers $W=\left\{w_{i}\right\}$, and a target $V$. We construct a corresponding two user Min-MA instance as follows. For every $w_{i}$, we construct a subcarrier $i$ with a ratepower function, which is the same for both users. Specifically, the power for rate 0 is 0 , the power for rate $w_{i}$ is $\frac{P}{|W|}$, the power for rate $w_{i}+\frac{1}{|W|}$ is $P$, and it is strictly increasing.

\footnotetext{
${ }^{2} \mathrm{~A}$ rate-power function for user $i$ and subcarrier $j$ combination describes the relation between required transmit power and the rate loaded on subcarrier $j$, under user $i$ 's BER constraint. Thus, the influence of channel gain is also included in this function. An example rate-power function for QAM can be found in [4].
}

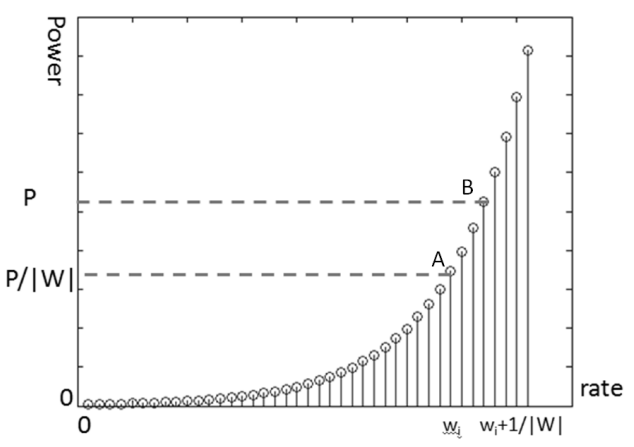

Fig. 1. An example rate-power curve. (Note: For illustration purpose only.)

An example plot of this constructed rate-power equation is in Figure 1.

We claim that Subset Sum has a satisfying solution if and only if Min-MA has an assignment, which can satisfy two users with rate requests $V$ and $\left(\sum_{i=1}^{m} w_{i}-V\right)$ and the total power consumption is no more than $P$.

Suppose Subset Sum has a solution such that the sum of the subset $S$ is exactly $V$. If we allocate every corresponding subcarrier in $S$ to one user, all the remaining subcarriers to the other user, and load every subcarrier with rate $w_{i}$, then this assignment would be a satisfying solution to Min-MA. On the other hand, if $S$ does not exist, then no subset can give us a sum exactly $V$. Because every $w_{i}$ is a natural number, the difference of sum between any subset and $V$ must be no less than 1. In addition, the largest number of subcarrier a user can be allocated is $|W|-1$. Consequently, one of the two users has to load at least one of the subcarrier assigned to him/her with rate higher than $w_{i}+\frac{1}{|W|}$, which implies the maximal individual power consumption higher than $P$, thus making the total power consumption also higher than $P$.

\section{Implications}

In the above proof, we have demonstrated that every Subset Sum instance can be transformed to a two-user instance of Min-MA, that both users experience the same channel conditions. Because the transformed instances are only a subset of Min-MA and it is NP-hard already, a more general case, i.e. more than two users and users may experience different channel conditions with respect to the the same subcarrier, must also be NP-hard. In addition, because the maximal user's transmit power also exceeds $P$ if and only if overall power exceeds P, MinMax-MA is NP-hard as well. 


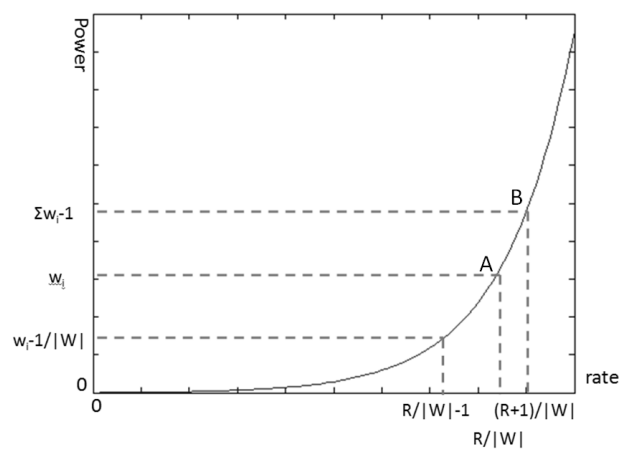

Fig. 2. An example rate-power curve of a constructed subcarrier in Max-RA.

In some papers, like [15], it is assumed that every subcarrier has to be allocated with equal power for Min-MA. In the above proof, the optimal solution for the transformed instance loads every subcarrier with identical power. Therefore, even Min-MA with extra identical rate constraint, it is still NP-hard. In other words, loading identical power on each subcarrier does not help reduce computational complexity.

The decision version of Max-RA can be stated as:

Problem 2: Given a system power budget $P$, nondecreasing rate-power functions for $n$ users and $m$ subcarriers combination, and $m>n$, does there exist a set of subcarrier and user assignment with power allocation on individual subcarrier, such that the overall transmit rate is at least $R$, power budget $P$ is honored, and no subcarrier is allocated to more than 1 user?

Consider the rate-power functions of the transformed MaxRA instance have the following properties: (1) the power for rate 0 is $0,(2)$ the power for rate $\frac{R}{|W|}-1$ is $w_{i}-\frac{1}{|W|}$, (3) the power for rate $\frac{R}{|W|}$ is $w_{i}$, (4) the power for rate $\frac{R+1}{|W|}$ is $\sum_{i=1}^{m} w_{i}-1$, (5) $\sum_{i=1}^{m} w_{i}=P$, and (6) they are strictly increasing. An example of these functions is plotted in Figure 2. Consequently, a line by line similar proof as above can show that Max-RA is NP-hard. With similar reasoning as MinMax-MA is NP-hard, it is straightforward to demonstrate MaxMin-RA is NP-hard.

Although all the above conclusions are made for the single cell situation, i.e. all users are served by the same base station, since the multi-cell scenario is a superset of single cell setting, multi-cell versions of Min-MA, MinMax-MA, Max-RA and MaxMin-RA are also NP-hard.

Because the above proof does not require convexity of rate-power functions, even if the rate-power functions are continuous and convex, as used in [3], [11] and [4], the NPhardness conclusion for Min-MA, MinMax-MA, Max-RA and MaxMin-RA still holds. Moreover, since we do not assume the continuity of rate-power functions used in the proof, both continuous and discrete version of these problems are NPhard.

\section{IN-APPROXIMABILITY AND PERFORMANCE BOUND}

\section{A. Proof Strategy}

We first state the definition of $\alpha$-Approximation Algorithm.
Definition 2: If we denote the optimal solution and the solution generated by a $\alpha$-approximation algorithm of an optimization problem as $O P T$ and $S O L$, respectively, then the ratio $\frac{S O L}{O P T}$ is always no more than $\alpha$ for a minimization problem, and no less than $\alpha$ for a maximization problem.

In short, the constant factor $\alpha$ is a guaranteed performance bound for the solutions generated by an approximation algorithm.

The strategy we use in the following proof is called gap introducing reduction [16]. The basic idea is, we first do polynomial reduction to transform an arbitrary instance of Subset Sum to a special case of problems of interest. If there exists an polynomial time approximation algorithm for our problems, then we can use it to determine the solutions of Subset Sum problem in polynomial time, which leads to a contradiction.

\section{B. In-approximability Proof}

Theorem 2: Achieving an approximation ratio $\alpha, \forall \alpha>1$ for Min-MA is NP-hard.

Proof: The process of doing polynomial reduction to transform an arbitrary instance of Subset Sum to Min-MA is line by line similar as prior proof, except that the power for rate $w_{i}+\frac{1}{|W|}$ is $\alpha P$ for all rate-power functions. Again, these rate-power functions are strictly increasing.

We claim that Subset Sum has a satisfying solution if and only if a polynomial time $\alpha$-approximation algorithm of MinMA generates an solution with total power consumption at most $\alpha P$.

Suppose the input instance of Subset Sum has a solution such that the sum of the subset $S$ is exactly $V$, then we know the optimal solution for the transformed instance of Min-MA is no more than $P$. Consequently, an $\alpha$-approximation algorithm of Min-MA will generate a solution that the objective value is at most $\alpha P$.

On the other hand, if the input instance of Subset Sum does not have a satisfying solution, then we know no subset in $W$ can have sum equal to $V$. Because every $w_{i}$ is a natural number, the difference between the sum of any subset and $V$ must be no less than 1 . In addition, the largest number of subcarrier a user can be allocated is $|W|-1$. Consequently, one of the two users has to load at least one of the subcarrier assigned to him/her with rate higher than $w_{i}+\frac{1}{|W|}$. Therefore the minimum overall transmit power consumption for the transformed instance is higher than $\alpha P$, which implies the solution provided by an $\alpha$-approximation algorithm must also be higher than $\alpha P$.

In sum, by doing polynomial reduction to transform an arbitrary instance of Subset Sum to a Min-MA instance, we can determine the satisfiability of Subset Sum by using a polynomial time $\alpha$-approximation algorithm of Min-MA as follows: whenever the objective value generated by the $\alpha$-approximation algorithm is no more than $\alpha P$, we know the input instance of Subset Sum has a satisfying solution; otherwise, the input instance of Subset Sum cannot be satisfied.

For Max-RA, we have a different conclusion:

Theorem 3: Achieving an approximation ratio $\frac{1}{m-1}$ for Max-RA, where $m$ is the number of available subcarriers, 
is NP-hard.

Proof: Consider the polynomial reduction of Theorem 2 with the following changes for every rate-power function: (1) the power for rate $\frac{\alpha R-\frac{R+1}{|W|-1}}{|W|}$ is $w_{i}-\frac{1}{|W|}$, (2) the power for rate $\frac{R}{|W|}$ is $w_{i}$, (3) the power for rate $\frac{R+1}{|W|}$ is $\sum_{i=1}^{m} w_{i}$, (4) the power for rate 0 is 0 , and (5) all these rate-power functions are strictly increasing.

We claim that Subset Sum has a solution if and only if an $\alpha$-approximation algorithm of Max-RA gives a solution with overall transmit rate at least $\alpha R$.

Suppose Subset Sum has a satisfying solution such that the sum of a subset $S$ is exactly $V$, then we know the optimal solution for the transformed Max-RA instance is no less than $R$. Consequently, an $\alpha$-approximation algorithm of Max-RA will give a solution where the overall transmit rate is no less than $\alpha R$.

If the input Subset Sum instance does not exist a satisfying solution, then no subset in $W$ can give us a sum exactly $V$. Because every $w_{i}$ is a natural number, the difference between sum of any subset and the target value $V$ must be no less than 1. In addition, the largest number of subcarrier a user can be allocated is $|W|-1$, and the highest power budget a user can have is $\sum w_{i}-1$. Consequently, one of the two users has to load one of the subcarrier assigned to him/her with power at most $w_{i}-\frac{1}{|W|}$. On the other hand, the other user can load at most one of the sub-carrier allocated to him/her with power no higher then $\sum w_{i}-1$, thus the overall transmit rate these two users can achieve must be less than $\alpha R$, and so is the output of the $\alpha$-approximation algorithm.

In short, if an $\alpha$-approximation algorithm of Max-RA generates a solution with overall transmit rate no less than $\alpha R$ for the transformed instance, then we know the input Subset Sum instance has a satisfying solution; otherwise, there exists no subset in $W$ such that its sum equals to $V$. Thus, we always can use a polynomial time $\alpha$-apprixmiation algorithm of Max-RA to determine the satisfiability of any instance of Subset Sum in polynomial time, which is a contradiction.

Note that, due to the non-decreasing property of every rate-power function, we need $\frac{\alpha R-\frac{R+1}{|W|-1}}{|W|}>0$, which implies $\alpha>\frac{R+1}{(|W|-1) R}$. When the input instance is large, i.e. $|W|$ is large, this value approaches $\frac{1}{|W|-1}=\frac{1}{m-1}$, thus completes the proof.

\section{Implications}

Because approximating Min-MA within the factor $\alpha, \forall \alpha>$ 1 is NP-hard, it is impossible for any polynomial time algorithm designed for Min-MA to have a guaranteed performance bound, if $P \neq N P$. In other words, every polynomial time algorithm designed for Min-MA may generate arbitrarily far from optima solutions, unless $P=N P$. For Max-RA, because of the hard-to-achieve approximation ratio $\frac{1}{m-1}$, where $m$ is the number of available subcarriers, no polynomial time algorithm can have an approximation ratio tighter or equal to $\frac{1}{m-1}$, unless $P=N P$.

In the proof of Theorem 2, we notice that the maximal user's transmit power also exceeds $\alpha P$ if and only if the overall power exceeds $\alpha P$, thus achieving an approximation ratio $\alpha, \forall \alpha>1$ for MinMax-MA, is NP-hard as well. On the other hand, in the proof of Theorem 3, the minimal user's transmit rate also exceeds $\alpha R$ if and only if the overall rate exceeds $\alpha R$, thus achieving an approximation ratio $\frac{1}{m-1}$ for MaxMin-RA, is NP-hard as well.

Note that, in the proof of Theorem 2, the optimal solution for the transformed instance loads every subcarrier with identical rate. Therefore, for Min-MA and MinMax-MA with extra identical rate constraint, such as [5], they are still impossible to achieve a constant factor bound $\alpha, \forall \alpha>1$, unless $P=N P$. Similarly, the variant of Max-RA and MaxMin-RA, which require every subcarrier assigned to the same user to be loaded with equal power, cannot have an approximation ratio of $\frac{1}{m-1}$, if $P \neq N P$.

Using a similar reasoning as in prior section, multi-cell version of Min-MA and MinMax-MA cannot be approximated within the factor $\alpha, \forall \alpha>1$, and multi-cell version of Max$R A$ and MaxMin-RA cannot be approximated within the factor $\frac{1}{m-1}$, if $P \neq N P$.

By observing the above proofs, we notice that the most challenging part of dealing with the above subcarrier allocation problems is how to optimally Allocate disjoint set of Subcarriers to users (We refer this problem to as $A S$.). Note that, even if the optimal number of subcarriers that each user gets assigned is known, it still does not help reduce algorithmic difficulty of MA and RA related problems. If AS can be solved optimally in polynomial time, then adopting water-filling policy, which can be accomplished in polynomial time, can solve the whole problem optimally. More specifically, there are two issues make AS difficult to handle: (1) no subcarrier can be shared, and (2) every user's request needs to be honored. If the first assumption, i.e. exclusive subcarrier usage, is relaxed, then using convex programming can achieve optimality of Min-MA in polynomial time [11]. If the second assumption, i.e. every rate request is satisfied, is relaxed, then using dynamic programming, which is pseudopolynomial time, can always solve Min-MA optimally [17]. In addition, many fully polynomial time approximation scheme (FPTAS) designed for Multi-choice Knapsack [16] can be applied directly and the approximation factor can be arbitrarily close to 1 at the expense of running time [17].

Again, even the convexity and/or continuity of rate-power functions are assumed, the hard-to-approximate factors stated above still hold.

\section{Worst Case Scenarios}

Because of the NP-hardness and in-approximability conclusions, it will be helpful to understand when a polynomial time algorithm may perform undesirably.

By observing the proofs of Theorem 2 and 3, we notice that while the rate difference between point $\mathrm{A}$ and $\mathrm{B}$ in Figure 1 is small, the power difference between these two points is large, the same holds true in Figure 2. There are couple of situations which may make a rate-power function have drastic increase. First, when the number of available coding/modulation schemes are fixed, the highest rate that can be supported is fixed. Therefore, the rate-power function beyond the maximum support rate directly jumps to infinite power for this subcarrier. Alternatively, if the power that can be loaded onto a subcarrier is fixed, then it will also lead to 
a rate-power function that has drastic increase. In addition, when deep fading occurs, the rate-power function also has a steep increase in power. In general, if an algorithm allocates an improper subset of subcarriers to a user, and this user is forced to operate in the steep increase region, then the system objective will be deteriorated drastically. In other words, the impact of imperfect AS solutions can be largely amplified by rate-power functions which have steep increase region.

The following situations may lead to undesirable solution quality for every polynomial algorithm:

- The number of users are close to the number of available subcarriers. Because subcarriers cannot be shared by multiple users, the number of subcarriers a user can get is small. Thus, the system objective is highly influenced by the solution quality of AS generated by algorithms. However, AS is NP-hard by itself, which implies every polynomial time algorithm may perform poorly in this situation.

- Users' requests are close to system capacity. In practice, only a fixed number of coding/modulation schemes are available. If sum of users' requests is close to the highest possible rate that can be supported by the system, then the system objective will be highly related to the solution quality of AS. Therefore, any nonoptimal user-subcarrier assignment may lead to degraded system performance. Note that, the situation when most subcarriers are in deep fading has the same effect as high users' requests.

- Users experience high variance of channel gain. When the variance of channel gain is high, some deep fading subcarriers may be utilized by imperfect user-subcarrier assignment. Thus, system objectives depend on quality of AS, which implies the risk of poor system performance again. The same observation also applies to the scenario that some subcarriers have high gains, but remaining subcarriers are in deep fading for all users, i.e. there is a lack of multi-user diversity.

- Frequency diversity is low. Because every user request needs to be honored, if some users experience deep fading on every subcarrier, i.e. there is a lack of frequency diversity, then any subcarrier-user assignment that is different from optimal ones may lead to tremendous deterioration of system objective.

Note that, although we have shown the inapproximable/hard-to-approximate conclusions, it does not necessarily mean that algorithms will always perform terribly in the above scenarios. But at the same time, we are arguing that the optimal solutions cannot be guaranteed by any polynomial time algorithm. Every polynomial time algorithm is likely to perform poorly under these challenging conditions, where the worst case solution quality may be far away from optimum.

\section{CONCLUSIONS}

We rigorously demonstrate the NP-hardness of a family of subcarrier allocation problems. More importantly, we show the fundamental limits of all polynomial time algorithms: for Min-MA and MinMax-MA, every polynomial time algorithm may perform arbitrarily far from optimal solutions, unless
$P=N P$; for Max-RA and MaxMin-RA, no polynomial time algorithm can have a guaranteed approximation ratio better than $\frac{1}{m-1}$, where $m$ is the number of available subcarriers, unless $P=N P$. We also identify qualitatively the worst case scenarios where all polynomial time algorithm may generate poor solution quality.

\section{ACKNOWLEDGMENT}

This work was supported in part by NSF through grants numbered CNS-0347621 and CNS-0627028, and through funding from Sprint.

\section{REFERENCES}

[1] IEEE, "802.16: Worldwid interoperability for microwave access (wimax)," December 2001.

[2] —_ "802.11g: Lan/man standards committee," June 2003.

[3] D. Kivanc, G. Li, and H. Liu, "Computationally efficient bandwidth allocation and power control for ofdma," IEEE Trans. on Wireless Communications, vol. 2, 2003.

[4] C. Wong and R. Cheng, "Multiuser ofdm with adaptive subcarrier, bit, power allocation," IEEE JSAC, vol. 17, no. 10, October 1999.

[5] I. Kim, I. Park, and Y. Lee, "Use of linear programming for dynamic subcarrier and bit allocation in multiuser ofdm," IEEE Trans. on Vehicular Technology, 2006.

[6] A. Abrardo, A. Alessio, P. Detti, and M. Moretti, "Radio resource alllocation problems for ofdma celluar systems," Computers and Operating Research, vol. 36, 2009.

[7] M.Bohge, J. Gross, and A. Wolisz, "A new optimization model for dynamic power and sub-carrier allocations in packet-centric ofdma cells," Proc. Of 11th International OFDM-Workshop, August 2006.

[8] Z. Shen, J. Andrews, and B. Evans, "Adaptive resource allocation in multiuser ofdm systems with proportional rate constraints," IEEE Transactions on Wireless Communications, vol. 4, November 2005.

[9] T. Cover and J. Thomas, "Elements of information theory," 1991.

[10] J. Kleinberg and E. Tardos, Algorithm Design. Addison-Wesley, 2006.

[11] W. Rhee and J. Cioffi, "Increase in capacity of multiuser ofdm system using dynamic subsubcarrier allocation," Proceedings of VTC, 2000.

[12] J. Jang and K. Lee, "Transmit power adaptation for multiuser ofdm systems," IEEE JSAC, vol. 21, 2003.

[13] J. Campello, "Discrete bit loading for multicarrier modulation systems," Stanfor Univ. Ph.D. dissertation, 1999.

[14] T. Alen, A. Madhukumar, and F. Chin, "Capacity enhancement of a multi-user ofdm system using dynamic frequency allocation," IEEE Proc. of WCNC 2003, 2003.

[15] G. Li and H. Liu, "Downlink radio resource allocation for multi-cell ofdma system," IEEE Trans. on Wireless Communications, vol. 5, 2006.

[16] V. Vazirani, Approximation algorithms. Springer, 2003.

[17] R. Cohen and L. Katzir, "Computational analysis and efficient algorithms for micro and macro ofdma scheduling," IEEE Proc. of Infocom, 2008. 